\title{
Reply to Betts et al. "When are hypotheses useful in ecology and conservation?"
}

\author{
Meredith Root-Bernstein ${ }^{1}$ \\ ${ }^{1} \mathrm{CNRS}$
}

April 22, 2021

Reply to Betts et al. "When are hypotheses useful in ecology and conservation?"

Meredith Root-Bernstein ${ }^{1,2,3}$

1. UMR CESCO, CNRS, Muséum National d'Histoire Naturelle, Paris, France

2. Center of Applied Ecology and Sustainability, Santiago, Chile

3. Institute of Ecology and Biodiversity, Santiago, Chile

Words: 2045

It is difficult to disagree with Betts et al. (2021) when they claim that hypotheses are often useful but sometimes not necessary. The difficulty with Betts et al. does not lie with any of their individual points, but rather with the lack of a clear argument giving them structure. This is not just a critique of style. It is relevant because it is an example of what I think is the real problem in ecological research. In my view, the lack of hypotheses in ecology and conservation is not just about the rise of big data approaches, or the documentation of applied work. More generally, I argue that the low use of hypotheses reflects the failure of ecology and conservation to value and develop discipline-specific forms of argument, logic and reasoning. I first address the particular nature of the hypothesis as an argument form, and then the question of whether there are specifically ecological argument forms. Finally I argue that we need a broad set of arguments and logics suitable to the broad set of phenomona in ecology, and that hypotheses are usually derived from non-hypothetico-deductive reasoning and logic. If we want more or better hypotheses, we need more and better forms of non-hypothetico-deductive ecological reasoning.

A hypothesis is a form of argument structured so that it can be answered in only one of two ways: rejection or non-rejection. Hypotheses are also characterized by particular ways of framing questions that are considered legitimate, interesting, or elegant, which varies by the discipline or subject matter. I will illustrate my points about the need for forms of argument that fit a subject matter with the Betts et al. paper itself. Betts et al. present their argument about why ecologists should use hypotheses in the form of a couple of hypotheses, the predictions of which they test in a hypothetico-deductive manner on quantitative data using statistical reasoning. They structure their hypothesis as though it were an evolutionary argument: they identify potential discrete individual benefits of adopting a behaviour within a specific environment.

Hypotheses ask narrow questions

Two things strike me about this way of arguing about hypothesis use. First of all, by casting scientists as rational actors who are passively being "selected on" through their responses to cues in their professional environment, this argument circumvents any discussion of how science knowledge is formed, or about how scienceconventions and social structures are negotiated, and the role of hypotheses in these processes. These fundamental issues simply cannot be addressed with this reasoning. This characterisation of science and scientists in the framing of the hypotheses is certainly questionable. In this case the problems of the underlying 
theoretical framing dovetail with the rush to frame the question as hypothetico-deductive. The limitation to yes-no type answers in response to hypotheses excludes, for example, answers of the type that raise new questions. These might include, for example, "in fact we find that hypothesis use is a rather late step in knowledge creation within the scientific process and most of ecology research programs are not in that stage" or "in fact we find that hypothesis use is suitable for certain types of questions, and ecological research is not oriented towards such questions" or "in fact we find that anonymous reviewers are more likely to reject papers that attempt to test predictions than those that do not" or whatever other issues might emerge from a less narrow approach. Indeed, arguably Betts et al. is a case where the question has been narrowed down to a hypothesis with a yes-no answer much too early - so early as to eliminate the consideration of a rich set of concerns and factors. The issue of how scientists construct knowledge deserves much more exploration before carefully evaluating which, if any, questions can be further refined into questions suitable for a hypothetico-deductive approach.

At the same time, one might object that ecology and conservation scientists are not obliged to structure their research questions by drawing on epistemology (the study of how we know and come to know things) and Science and Technology Studies because these are other disciplines, and no-one is obligated to be interdisciplinary (but see Boon \& Van Baalen 2019). Multiple disciplines exist since (to simplify), historically, particular methods and approaches have been more generative for particular subjects than others. This same consideration is pertinent for within-disciplinary questions as well. Just because we have historically drawn an arbitrary line around non-human living things and designated them as "ecology" and thus subject to the hypothetico-deductive approach, this does not mean that hypothetico-deductive approaches are always the best means to understand all aspects of ecological phenomena. My counter-claim is that we need to be "inter-methodological" or plurimethodological even if we are not being interdisciplinary. I will expand on this briefly below.

\section{Are there ecological argument forms?}

The second reflection that Betts et al.'s argument structure raises for me is especially pertinent to ecology as a science. I am struck that the form of argument that Betts et al. present, in which the scientist is a rational actor passively selected on by his environment, is drawn not from ecology, but from evolutionary biology. If there were a standard argument form about, say, ecological succession that the authors had drawn on, using it here could be a clever and ironic meta-argument (if still not well adapted to the question at hand). However, it appears to me that there are no standard forms of argument about ecological phenomena. The closest thing I can think of is the idea that you derive hypotheses by extending a mechanism observed at a small scale across time and space. However, the qualities of various forms of reasoning about pattern interactions or up- and down-scalings over time and space are not well established. Ecology has systematically undervalued all forms of non-statistical reasoning (statistical reasoning also requires further discussion, e.g. Amrhein et al. 2019). Reasoning across time and space is, however, about more than statistics. It should also involve judgements about when, where and why certain kinds of scaling phenomena occur.

For example, we often use emergent-phenomena reasoning to explain the interactions of monospecific groups, but rarely heterospecific groups and never the assembly of species communities - why? We often use attractor states to explain degradation, but not to explain community assembly or interactions of monspecific groupswhy? If I were to propose that we model degradation as being caused by an emergent property of multiple species interactions, or that we think of termite mounds as formed by moving towards structural attractors, these might sound "wrong", but I posit that ecologists have developed very few logical or rhetorical resources for arguing for or against such proposals. Some ecologists might object here that the argument behind the model is irrelevant because the model either corresponds to (or predicts) empirical observations, or not. However, I am willing to bet that by relabelling the variables in a model of termite nest building or a model of degradation, the phenomenon they are supposed to be representing could be switched such that the model of degradation would now explain the canalization of the nest shape into a typical form, while the model of the termite nest would explain the reordering of species into an irreversible pattern. It is thus the model that is relatively irrelevant - only the argument specifies what it is that we claim to be explaining, and thus 
how we evaluate the model.

Betts et al., like most ecologists, rely entirely on statistical reasoning as their analytical approch to answering their questions, and thus structure the core of their argument in the form of predictions about whether hypothesis use has increased or decreased. This is, frankly, the most boring possible question that could be asked about hypothesis use. Other things one would like ask questions about are, at least in my opinion, the things I am talking about here. However, the question of whether hypothesis use has gone up or down is also, I recognise, one of the only questions that can be tested using available quantitative data and formulated as something that can be answered 'yes' or 'no' through statistics. Betts et al. thus provides a demonstration of how a focus on hypothetico-deductive reasoning can limit researchers to asking and answering uninteresting, overly simplifed questions.

\section{Hypothesis use in the context of other forms of reasoning and logic in ecology}

While I agree with Betts et al.'s points about how hypotheses are excellent for being explicit, thinking through multiple possibilities, and avoiding your own biases, I cannot agree with what I take to be their argument that the hypothetico-deductive process is the culmination of science practice. In general, epistemology, like the ecological world itself that is our subject, is heterogenous, varying, and complex (see Liebenberg 2013). As I indicated above, our disciplinary line-drawing around living things does not mean that they are a uniform collection of phenomena that all behave in pre-identified ways. The identification of problems, and the reasoning approaches needed to answer questions about those problems, works well when it mirrors the heterogenous, varying, and interacting structure of living systems, through the use of a mix of multiple forms of reasoning, argumentation and logic. Hypothetico-deductive methods are of course a hallmark of science, and are powerful if used intelligently (following many of the suggestions in Betts et al.), and if not over-applied to every question. The real question is not whether to use hypotheses, but how to recognise and develop a question that can be best answered with a hypothetico-deductive process. This is not an easy question to answer, and although many experienced ecologists and other scientists develop a sense of how to track down and corner a good hypothesis, this is something we should discuss more in order to develop disciplinary aesthetics or styles around hypothesis formation (Root-Bernstein 2002; Eastwood et al. 2013).

The equally important corrolary to this is how to recognise a question that can best be answered with other forms of reasoning, logic, and argument. This is especially true if we consider the need to engage in interdisciplinary research to more fully understand socioecological systems (Root-Bernstein 2016), or the desireability of engaging with indigenous and local knowledge (ILK) in conservation and ecology projects (Wheeler \& Root-Bernstein 2020). Having a range of forms of reasoning, logic, and argumentation proper to ecology that go beyond statistical answers to questions posed as hypotheses will provide multiple avenues for communication and collaboration. There is also, in my view, a benefit to the explicit recognition that ecological science has (or should have) its own set of arguments, logic and reasoning, and that it has (or should have) styles and aesthetics of different qualities of reasoning. It is relatively more difficult to collaborate with ILK holders if your only account for why you use the hypothetico-deductive method is that it directly reveals the true structure of the universe. When we can discuss our aesthetics and logics around good hypothesis use, we open the door to being relativistic in a constructive/ productive way.

\section{We need other forms of logic and reasoning to develop hypotheses}

Finally, and critically, I would suggest that hypotheses are often derived through reasoning that is not hypothetico-deductive in nature (Root-Bernstein \& Root-Bernstein 1999). One reason why hypothesis use may be low in ecology is because these other forms of reasoning needed to create good hypotheses are undervalued and crowded out. Ecology and Evolution is one of only a handful of journals that have a specific "Hypothesis" article format. In my experience, an interesting and novel hypothesis in ecology, socioecology, the ecology-evolution interface, or conservation, can take multiple pages to fully reason through, justify, and render precise, as different forms of data (cross-disciplinary, observational, inductively derived, ILK, experimental, etc.) are woven together. Darwin required several entire books to work out his ideas. In contemporary ecology, the people who write book-length research texts are mainly eminent scientists with 
international reputations. These should not be the only people, or the only phases in one's career, dedicated to ecological reasoning. We all need experience with other forms of logic and reasoning to construct good hypotheses. Thus giving space to the reasoning required to develop interesting and novel hypotheses can also promote the development of these multiple forms of non-hypothetico-deductive argumentation, reasoning, and logic suited to ecological subjects.

Data Accessibility Statement:

There is no data to make available.

References:

Amrhein, V., Greenland, S., \& McShane, B. (2019). Scientists rise up against statistical significance. Nature, 567, 3015-307.

Betts, M. G., Hadley, A. S., Frey, D. W., Frey, S. J., Gannon, D., Harris, S. H., .. \& Zárrate-Charry, D. (2021). When are hypotheses useful in ecology and evolution?. Ecology and Evolution .

Boon, M., \& Van Baalen, S. (2019). Epistemology for interdisciplinary research-shifting philosophical paradigms of science. European journal for philosophy of science, 9 (1), 1-28.

Eastwood, J. L., Sadler, T. D., Sherwood, R. D., \& Schlegel, W. M. (2013). Students' participation in an interdisciplinary, socioscientific issues based undergraduate human biology major and their understanding of scientific inquiry. Research in Science Education , 43 (3), 1051-1078.

Liebenberg, L. (2013). The origin of science . Cape Town: CyberTracker.

Root-Bernstein, M. (2016). Personal reflections on natural history as common ground for interdisciplinary multispecies socio-ecological research. Geo : Geography and Environment 3(1), e00015

Root-Bernstein, R. S. (2002). Aesthetic cognition. International Studies in the Philosophy of Science, 16(1), 61-77.

Root-Bernstein, R., \& Root-Bernstein, M. (1999). Sparks of genius: The 13 thinking tools of the world's most creative people. New York, Houghton Mifflin.

Wheeler, H, \& Root-Bernstein, M. (2020). Introduction to special feature: Integrating indigenous and local knowledge into conservation science and decision-making. Journal of Applied Ecology 57, 1634-1643. 